\title{
DISTRIBUCIÓN Y NUTRICIÓN MINERAL DE Salicornia ramosissima J. Woods, Salicornia europaea L. $Y$ Salicornia dolichostachya Moss. EN EL ESTUARIO DE LOS RÍOS ODIEL Y TINTO (Huelva, SO España)
}

\author{
E. Figueroa*; J. Jiménez-Nieva*; J. Carranza* y C. González Vilches** \\ * Departamento de Ecología. Universidad de Sevilla. Apartado 1095 \\ ** Departamento de Química Inorgánica. Facultad de Farmacia. Universidad de Sevilla.
}

Palabras clave: Salicornia distribution, Salicornia mineral contents.

\begin{abstract}
DISTRIBUTION AND MINERAL NUTRITION IN SALICORNIA RAMOSISSIMA, SALICORNIA EUROPAEA AND SALICORNIA DOLICHOSTACHYA IN ODIEL AND TINTO MARCHES (SW SPAIN)

S. ramosissima, S. europaea and S. dolichostachya grow in sand and mud deposits without space limitation and high tidal influence. Mineral contents in leaves shows differences due to soil and flooding. We discuss the role of Salicornia in the estuary food chain.
\end{abstract}

\section{INTRODUCCIÓN}

Las marismas del estuario de los ríos Odiel y Tinto (alrededor de $10.000 \mathrm{Ha}$ ) constituyen uno de los ecosistemas húmedos más ricosdel litoral peninsular. En ellas se encuentran todos los estadios sucesionales de marismas, generándose una alta diversidad de hábitats (Figueroa, 1987). Recientemente han sido incluidas en la red europea de Reservas Biogenéticas (Dijkema, 1987).

El componente anual más importante de la vegetación de este sistema viene dado por las especies Salicornia ramosissima J. Woods, S. europaea L. y S. dolichosfachya Moss (se sigue la nomenclatura de Valdés et al., 1987).

Limnetica, 3 (2): 307-310 (1987)

(C) Asociaaón Española de Limnología, Madrid, Spain
Las diferentes especies del género están siendo muy estudiadas a nivel mundial por ser uno de los pocos que muestran en marismas taxones anuales en un marco general de perennes, sufrir una fuerte selección natural debido a su carácter anual, ser especies colonizadoras y, debido a su elevada producción primaria, suministrar una alta cantidad de detritos (Jefferies et al., 1979; Davy y Smith, 1985; Figueroa et al., 1987).

En el presente estudio se discute la distribución espacial y temporal de las tres especies, aportándose datos de su contenido mineral en la porción aérea, donde se detectan diferencias entre las tres, relacionablescon sus sustratos respectivos. 


\section{MATERIAL Y MÉTODOS}

Durante 1986 y 1987 se ha estudiado la distribución espacio-temporal de las tres especies mediante una red de 70 puntos de muestreo que abarcan toda la variabilidad geomorfológica de estas marismas donde se encuentran las especies mencionadas del género Salicornia L. En tres puntos seleccionadospor ser representativos para cada una de las tres especies, esteros fangosos de gran recorrido de marea (conSalicornia ramosissi$m a)$, depósitos arenosos recientes con alta incidencia mareal (conSalicornia europaea)y marisma alta con bajaincidencia mareal (conSalicornia dolichostachya) se tomaron muestras de la porción aérea de cada una. Limpiadas y secadas eran sometidas a una serie de análisis $(\mathrm{Na}, \mathrm{K}, \mathrm{Ca}, \mathrm{Mg}, \mathrm{Fe}, \mathrm{Co}$, $\mathrm{Cu}, \mathrm{Zn}, \mathrm{Mn}$ y Ni) mediante espectrometría de absorción en un aparato Perkin Elmer Modelo 2380. Se analizaron además N,P y B, mediante el método Kjeldahl, colonmetna con metavanadato y colorimeíría con quinalizarina, respectivamente.

Al final de la primavera se tomaron muestras de suelo en cada punto $(0-10 \mathrm{~cm})$, analizándose $\mathrm{Ca}, \mathrm{Mg}, \mathrm{Na}, \mathrm{K}, \mathrm{Fe}, \mathrm{Cu}, \mathrm{Mny} \mathrm{Zn}$ (segúnJackson,1982); $\mathrm{pH}$ mediante $\mathrm{pH}$-metro Crison en mezcla 1:1 en agua; conductividad eléctrica mediante conductivímetro Crison en mezcla 1:1 en invierno y verano.

\section{RESULTADOS}

Salicornia ramosissima resulta la especie más abundante, presentándose en poblacionesextensasde alta densidad en depósitosfangosos recientescon elevada incidencia mareal, entre la cota de marea baja muerta y de pleamar media. También en depósitos arenosos de la entrada del estuario y en zonas de marisma alta, afectadas por las mareas en pleamares de alto coeficiente o equinocciales. Esta última aparición es la menos frecuente. En depósitos arenosos se mezcla con Salicornia europaea, siendo difícil su separación.

Salicorniaeuropaease desarrolla típicamenteen depósitos arenosos, muy expuestos a mareas, de las zonas más jóvenes del estuario.
Salicornia dolichostachya, la menos abundante, se encuentra en zonas de marisma alta, desnuda y afectada por mareas dealto coeficiente $(>3.5 \mathrm{~m})$ o equinocciales; estas zonas están afectadas de encharcamiento mareal por lluvias.

Podemos encontrar estas tres especies todo el año en la marisma, ya que germinan las semillas pocodespués de su caída con las primeras lluvias (Figueroa et al., en preparación).

La germinación comienza en octubre en el caso de S. dolichostachya siendo el máximo de expresión en invierno. Esta especie florece y fructifica en julio, cerrando su ciclo dos meses antes que las otras dos. Esto puede ser debido al lugar en que vive afectada de elevadas salinidades en verano ( $>50 \mathrm{mS} / \mathrm{cm}$ ).

S. ramosissima y S. europaea florecen y fructifican fundamentalmente en octubre, pudiendo adelantarse en los enclaves más elevados de marismas a finales de agosto o septiembre. La lluvia de semillas es en noviembre, comenzando a germinar en forma masiva.

En la Tabla 1 se muestran los resultados de los análisis de suelos en enclaves típicos de las poblaciones de las tres especies. Destacan los valores bajos de sodio en las arenas, donde se desarrolla S. europaea, frente a los altos de otros enclaves. El pH es más elevado en los suelos con S. dolichostachya. Las mayores diferencias en conductividad eléctrica de invierno a verano se dan en las zonas más independizadasde la marea con valores estivales superiores a $40 \mathrm{mS} / \mathrm{cm}$ entre 0-10 cm donde se encuentran las raíces de estas especies. En los depósitos arenosos la diferencia de inviernoa veranoesde $5 \mathrm{mS} / \mathrm{cm}$. Laselevadas salinidades estivales de las zonas donde, fundamentalmente, se encuentra S. dolichostachyaequivalen a presiones osmóticas de disoluciones de 14 atm frentea los valores de 4 atm de los máximos estivales de las planicies mareales arenosas con $\mathrm{S}$. europaea. En muchos halófitosel declive de crecimiento comienza a partir de presiones osmóticas de 4 atm (Macke y Unga, 1971).

En la Tabla 2 se muestran los valores de contenido en nutrientes minerales en tallos suculentos de S. ramosissima y S. europaea en marzo (iniciodel crecimiento rápido), junio (finaldel crecimiento rápido) y octubre (floración, fructificación).Para 
Tabla I. Diferenciación química de los suelos donde se desarrollan cada una de las poblacionesde las tres especies de Salicmia.

Chemistry of the soils where each of the three Salicmia species develop.

\begin{tabular}{|c|c|c|c|}
\hline & $\begin{array}{l}\text { Suelos con } \mathrm{S} \text {. } \\
\text { ramosissim }\end{array}$ & $\begin{array}{l}\text { Sueloscon } \\
\text { S.europaea }\end{array}$ & $\begin{array}{l}\text { Suelos con } \mathrm{S} \text {. } \\
\text { dolichostachya }\end{array}$ \\
\hline Caomg/100g & 529 & 780 & 313 \\
\hline $\mathrm{MgO} "$ & 502 & 52 & 439 \\
\hline $\mathrm{Na}_{2} \mathrm{O} "$ & 3707 & 428 & 3803 \\
\hline $\mathrm{K}_{2} \mathrm{O} \quad "$ & 53 & 8 & 43 \\
\hline Fe ppm & 884 & 1117 & 683 \\
\hline Cuppm & 1352 & 12 & 492 \\
\hline Mn ppm & 185 & 793 & 290 \\
\hline Zn ppm & 1975 & 529 & 1342 \\
\hline $\mathrm{CE} \mathrm{mS} / \mathrm{cm}$ Invierno & 8 & 7 & 8 \\
\hline $\mathrm{CE} \mathrm{mS} / \mathrm{cm}$ Verano & 18 & 12 & 40 \\
\hline pH (inicioVerano) & 7.0 & 7.5 & 8.0 \\
\hline
\end{tabular}

S. dolichosfachya sólo se midieron los contenidos minerales del picode biomasa(julio).Esta especie muestra valores más bajos que las otras en casi todos los macro y micronutrientes. El contenido en metales pesados, hierro por ejemplo, es netamente más bajo. Esto puede ser debido al menor encharcamiento que sufren y al pH más alcalino del sedimento. El contenido en sodio y potasioes

Tabla II.Contenido mineral en parte aérea de S.ramosissima, S.europaea y S.dolichostachya Mineral contents of the aerial part of the three Salicornia species at different months

\begin{tabular}{|c|c|c|c|c|c|c|c|}
\hline \multirow[b]{3}{*}{$\mathrm{Na} \%$} & \multicolumn{3}{|c|}{$\begin{array}{l}\text { Salicornia } \\
\text { europaea }\end{array}$} & \multicolumn{2}{|c|}{$\begin{array}{c}\text { Salicomia } \\
\text { ramosissima }\end{array}$} & \multicolumn{2}{|c|}{$\begin{array}{r}\text { Salicomia } \\
\text { dolichastachya }\end{array}$} \\
\hline & Mano & Junio & Octub. & Marzo & Iunio & Octub. & Julio \\
\hline & 18.0 & 8.5 & 7.5 & 15.5 & 7.9 & 9.8 & 4.2 \\
\hline $\mathrm{K} \%$ & 3.6 & 1.5 & 1.3 & 3.2 & 1.2 & 1.3 & 1.1 \\
\hline $\mathrm{Ca} \%$ & 0.7 & 0.7 & 0.8 & 0.5 & 0.8 & 1.2 & 0.6 \\
\hline $\mathrm{Mg} \%$ & 1.3 & 1.1 & 1.0 & 1.1 & 1.0 & 1.1 & 0.7 \\
\hline $\mathrm{N} \%$ & 1.9 & 2.1 & 1.7 & 1.8 & 1.8 & 1.5 & 1.3 \\
\hline $\mathrm{P} \%$ & 0.2 & 0.2 & 0.2 & 0.4 & 0.4 & 0.3 & 0.2 \\
\hline B p p m & 33 & 15 & 12 & 76 & 75 & 70 & 55 \\
\hline Fe ppm & 2300 & 5400 & 6000 & 4400 & 30003 & 3400 & 300 \\
\hline Coppm & 0 & 0 & 0 & 14 & 16 & 15 & 10 \\
\hline Cuppm & 150 & 52 & 100 & 100 & 98 & 16.5 & 62 \\
\hline Znppm & 170 & 157 & 162 & 210 & 212 & 208 & 58 \\
\hline Mnppm & 170 & 22 & 30 & 60 & 16.5 & 35 & 147 \\
\hline $\mathrm{Ni}$ ppm & 510 & 65 & 72 & 38 & 38 & 33 & 15 \\
\hline
\end{tabular}

elevado en todos los casos, comoes característico de las especies hiperosmorreguladoras (Rozema efal., 1985).Los elevados valores de hierroencontrados en $S$. europaea y $S$. ramosissima inducen a pensar en una elevada traslocación de raíces a tallos. El manganeso seencuentra en mayor proporción en plantas de enclaves con menos sodio en el suelo. Algunos autores han puestode manifiestoque el cloruro sódico reduce la fitotoxicidad del manganeso en marismas (Singer y Havill, 1985). En enclaves más sometidos a encharcarnientosse estimula la entrada de hierro y manganeso por las raíces en $\mathrm{S}$. ramosissima y $\mathrm{S}$. europaea. En ninguna de las tres especiesla relación $\mathrm{Mn} / \mathrm{Fe}$ hace pensar que exista toxicidad de manganeso ya que no se alcanzan los valores cnticos de 10 a 30 (Rozemaetal.,1985 b). La razón N/Pincremen-
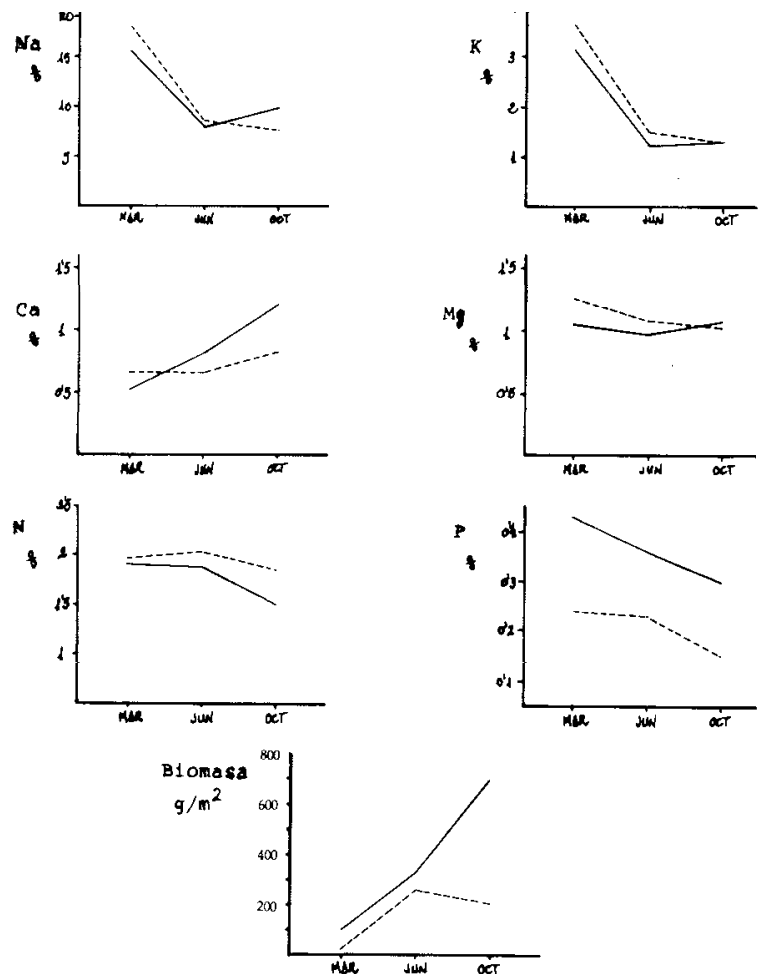

Figura 1.-Contenido mineral de la porciónaérea de Salicornia ramosissima (_- ) y S.europaea (--_--) a lo largo de su ciclo de vida. Se incluye un modelo de la evolución anual de la biomasa.

Elemental proportions of the aerial part of S. ramosissima (- - ) and S. europaea (--.--) during their lifecycle. A modelof their annual biomass development is included at the botton. 
ta desde final del invierno hasta el otoño, con valoresde8.0(marzo)all.3 (octubre)en S. europaea, y de 4.2 (marzo) a 5 (octubre) en S. ramosissima.

En la Figura 1 se muestra la variación del contenido en nutrientes minerales en estas dos últimasespeciesa lolargo del ciclodecrecimiento.Se incluye también en la figura las tendencias de incremento de biomasa de las mismas. Nitrógeno, fósforo, sodio y potasio disminuyen hacia el período de máxima biomasa e inicio de la senescencia. Resultaninteresantes loselevados valores de sodio al inicio del crecimiento.

\section{CONCLUSIONES}

S. ramosissima es la especie más abundante de las tres discutidas, ocupando la mayor parte de los espacios afectados por la marea que están desnudos de vegetación perenne de gran porte.S. europaea y S. dolichosfachyamuestranuna distribución más discreta, apareciendo respectivamente e n zonasmásarenosasmuy afectadaspor la marea y en zonas altas con menor incidencia mareal.

\section{Bibliografía}

DAVY, A. J. Y SMITH, H. (1985): "Population differentiation in thelife history characteristics of a salt marsh annual". Vegetatio, 61, 117-115.

DijkEMA, K. S. (1987): "Selection of salt marsh sites for the european network of biogenic reserves". Research lnstitute for Nature Manegement. Texel.

FiguEROA, E. (1987): "Ecología de las marismas del estuario de los ríos Odiel y Tinto (Huelva, SO España).Bases Científicas para la Proteccióndelos Humedales de España". Real Academia de Ciencias de Madrid.

Figueroa, E.; Fernandez-Palacios, J.; Carranza, J. y JIMÉNEZ-NiEva, J. (1987): "Tendencias de crecimientoenpoblacionesde SalicorniaramosissimaJ. Woods". Actas VIII Bienal de Historia Natural.

JACKSON, M. L. (1982): "Análisis químico de suelos". Omega. Barcelona. $4^{\mathrm{a}}$ edición. 662 pp.

Jefferies, R. L.; Davy, A. J. y Rudmik, T. (1979): "The growth strategies of coastal halophytes. Ecological
El contenido mineral de la porción aérea muestra marcadas diferencias entre ellas. Esta diferencia en contenido mineral refleja el sustrato donde viven, asícomo posiblescapacidadesmetabólicas propias. La elevada producción primaria neta de estas especies (alrededor de $700 \mathrm{~g} / \mathrm{m} 2$ ) hace que sean concentradoras y canalizadoras de nutrientes hacia el estuario. En el caso de S. ramosissima, la más abundante, se estima puede reciclar al estuario tras la senescencia, en el año siguiente, $\mathbf{1 0}$ $\mathrm{g} / \mathrm{m} 2$ de nitrógeno, $2 \mathrm{~g} / \mathrm{m} 2$ de fósforo y $9 \mathrm{~g} / \mathrm{m} 2$ de potasio. El papel de los halófitos de alta producción en la cadena detritívora es importante al suministrar nutrientes al estuario, por ellolas poblacionmes de Salicornia, además de su papel estabilizador de depósitos recientes, tienen un elevado valoren la producción total delosestuarios.

\section{AGRADECIMIENTOS}

La Comisión Asesora de InvestigaciónCientíficay Técnica(CAICYT) de la Juntade Andalucía subvencionó parcialmente esta investigación.
Processes in Coastal Environments". Blacwell. London. 243-268.

MACKE, A.J.; UnGAR, I. A. (1971):"'The effects of salinity on germination and early growth of Puccinellia nuttaeliana". Canadian Journal of Botany, 49, 515-520.

Rozema, J.; Bijwaard, P.; Prast, G. y BroekmanN, R. (1985): "Ecophysiological adaptations of coastal halophytes from foredunes and salt marshes". Vegetatio, 62, 499-521.

Rozema, J.; LuPPes, E. y BRoEKMANN, R. (1985b): “Differential response of salt marshes species to variation of iron and manganese". Vegetatio, 62,293-309.

Singer, C. E.; Havill, D. C. (1985): "Manganese as an ecological factor in salt marshes". Vegetatio, 62,287292.

Valdés, B.; Talavera, S. y Fernández-Galiano, E. (1987): "Flora Vascular de Andalucía Occidental". Tres tomos. Ketres Editora. Barcelona. 